\title{
Rethinking attentional development: reflexive and volitional orienting in children and adults
}

\section{Jelena Ristic ${ }^{1}$ and Alan Kingstone ${ }^{2}$}

1. Department of Psychology, University of California Santa Barbara, USA

2. Department of Psychology, University of British Columbia, Canada

\begin{abstract}
It is thought that a child takes the first 8 years of life to develop an adult-like volitional attention system. The data that support this belief, however, are based on studies that inadvertently measured a combination of volitional and reflexive attention, rather than volitional attention alone. What is immature then in children that are younger than 8 years of age? The volitional attention system or the manner that volitional and reflexive attention systems combine? We investigated this issue, with preschool and adult populations, by first isolating and then combining volitional and reflexive attention systems. Our results indicate that both volitional attention, and the way it combines with reflexive orienting, are immature in preschool children. We suggest that when volitional attention becomes adult-like its combination with reflexive attention will also become mature.
\end{abstract}

\section{Introduction}

The investigation of human attention is conducted using model tasks that enable one to isolate and manipulate mechanisms of cognitive processes within a laboratory environment. An excellent illustration of this approach is the Posner cuing task that has led to the development of several seminal theoretical models of attention (Posner, 1980; Jonides, 1981; Posner \& Petersen, 1990; Fan, McCandliss, Sommer, Raz \& Posner, 2002; Fan, McCandliss, Fossella, Flombaum \& Posner, 2005). In this framework, human attention is understood as a limitedcapacity process that can be controlled either reflexively by external stimuli in the environment or volitionally by internal changes in the goals and intentions of an individual. Two distinct versions of the cuing task have been developed. A peripheral cuing task (Posner, 1980) was designed to measure reflexive orienting, and a central arrow cuing task (Jonides, 1981) was designed to measure volitional orienting.

In the peripheral cuing task, the characteristics of reflexive attention are examined by requiring participants to detect a target light at a peripheral location that was, or was not, brightened. The brightening of a peripheral location, which is called the cue, does not predict where the target stimulus will appear. As a result, shorter response times (RTs) for targets appearing at the location of the cue are attributed to the reflexive orienting of spatial attention. The standard result is that average RT to a target at the cued location is facilitated when a target appears within $300-500 \mathrm{~ms}$ of the cue, after which RT is delayed at the cued location relative to an uncued location, reflecting the inhibition of return (IOR) phenomenon (Posner \& Cohen, 1984).

In the central arrow cuing task, the characteristics of volitional orienting are examined by requiring participants to detect a target light at a peripheral location that was, or was not, pointed at by a central arrow. The arrow, which is called the cue, does predict spatially where the target stimulus is likely to appear. Because spatial effects of the central arrow are assumed to occur only when the arrow is spatially predictive, the observed attention effects are attributed to volitional orienting of spatial attention (Jonides, 1981). The standard result is that RT to the target at the cued location is facilitated for all cue-target intervals exceeding $300 \mathrm{~ms}$ with no evidence of IOR emerging (e.g. Danziger \& Kingstone, 1999).

\section{Development of attention}

Age-appropriate modifications to the peripheral cuing task and the arrow cuing task have been applied to investigations of attentional development (e.g. Berger, Jones, Rothbart \& Posner, 2000). The data from these investigations suggest that reflexive and volitional attention exhibit different courses of development. While adult-like reflexive attention (early RT facilitation followed by IOR) is observed shortly after birth (e.g. Johnson, Posner \& Rothbart, 1991; Valenza, Simion \& Umiltà, 1994; MacPhearson, Klein \& Moore, 2003), adult-like volitional attention appears to emerge at a much later age (e.g. Brodeur \& Enns, 1997; Brodeur, 
Trick \& Enns, 1997; Goldberg, Maurer \& Lewis, 2001). Specifically, until 8 years of age, children can orient attention voluntarily towards a location indicated by a central predictive arrow cue but they are unable to sustain their volitional attention at that location.

This similarity between children and adults for reflexive orienting, and dissimilarity for volitional orienting, may stem from differential maturational rates of brain mechanisms implicated in controlling reflexive and volitional attention. Specifically, brain areas thought to subserve reflexive orienting, such as the superior colliculus and the parietal lobe, reach almost full functioning by 6 months of age whereas brain regions thought to play a critical role in controlling volitional orienting, such as the frontal lobe, do not fully mature until 8 years of age or more (Johnson, 2002, 2005; Corbetta \& Shulman, 2002).

\section{Rethinking the predictive central arrow cue model task}

Although it has long been believed that the central arrow cue task isolates and measures shifts in volitional spatial attention, recent studies conducted with preschool children and adults suggest reasons to question this classic result. In particular, it has recently been demonstrated that both adults and preschool children will orient their attention reflexively to a location indicated by an arrow, even when the arrow does not predict where a target is likely to appear (Ristic, Friesen \& Kingstone, 2002; Tipples, 2002). This finding raised the possibility that prior studies that have used central predictive arrows to measure volitional orienting may have unwittingly measured a combination of volitional and reflexive spatial orienting, rather than volitional orienting alone.

Ristic and Kingstone (2006) tested this hypothesis with adults. First, they confirmed that central number cues do not produce spatial orienting when they do not predict the location of a target stimulus. Second, number cues were made spatially predictive so as to engage volitional orienting in isolation. Third, they measured reflexive orienting by making the arrow cue nonpredictive. Finally, they measured performance when an arrow cue is made spatially predictive. The data indicated that the magnitude of the predictive arrow effect was significantly greater than either the magnitude of the isolated volitional attention effect obtained with predictive numbers or the magnitude of the isolated reflexive attention effect obtained with nonpredictive arrows. In fact, the magnitude of the predictive arrow effect was significantly greater than the sum of volitional and reflexive attention. Based on this result, Ristic and Kingstone concluded that the classic arrow cuing task engages both volitional and reflexive orienting, thus reflecting a superadditive interaction between volitional and reflexive attention.

\section{The present study}

The discovery that the predictive arrow task produces a superadditive orienting effect in adults sheds new light on the finding that children younger than 8 years of age do not demonstrate adult-like orienting effects in this task. In the past, this difference between adults and children was attributed to children not having an adult-like volitional attention system. However, given that for adults the task measures an interaction between volitional and reflexive attention, one must reconsider the interpretation placed on the children's data. Specifically, it is possible that children's immature performance on a predictive arrow cuing task reflects differences in how children and adults combine volitional and reflexive attention rather than developmental differences in volitional orienting alone. If this hypothesis is confirmed, the implication is that previous studies of attentional development may have mischaracterized the development of voluntary attention in childhood.

The goal of the present study was to examine the source of preschool age children's immature performance on the classic spatially predictive arrow task. Adopting Ristic and Kingstone's (2006) design, we measured reflexive and volitional orienting in isolation, and in combination in preschool children and adults. For both age groups, separate and combined effects of reflexive and volitional attention were acquired in four test conditions: (1) a Nonpredictive Arrow condition (NA), that triggers reflexive attention alone; (2) a Predictive Arrow (PA) condition, that can furnish both reflexive and volitional orienting; (3) a Nonpredictive Shape (NS) condition, that fails to trigger any spatial orienting confirming that the predictive shape condition is measuring volitional attention alone; and (4) a Predictive Shape (PS) condition, that engages volitional attention alone.

Anticipating the outcome, our results show that when reflexive and volitional orienting are isolated for preschool children, reflexive orienting is adult-like but volitional orienting is not. When these two forms of orienting are confounded in the classic predictive arrow task, reflexive and volitional attention effects combine in an additive manner. This additivity between attention systems observed for preschool children contrasts with the superadditive interaction observed for adults.

\section{Method}

\section{Participants}

Sixty-two (62) preschool children and 60 undergraduate adult students participated in the study. A failure to emphasize response speed (mean RT $>2 S D$ ) resulted in four children and three adults being excluded from the analysis. Detailed participant information is presented in Table 1.

\section{Apparatus, stimuli and design}

All stimuli were black line drawings shown on a white background using a 3200c Macintosh Power Book with 
Table 1 Participant information, response errors, interparticipant mean RTs and standard deviations for children and adults. Reported error rate represents an average of timed-out responses, incorrect key presses, and false alarms for each condition. * Since there were no true 'cued' and 'uncued' target conditions in the nonpredictive shape condition, corresponding numbers represent mean RTs for targets appearing on the left and right side locations for each SOA

\begin{tabular}{|c|c|c|c|c|c|c|c|c|}
\hline \multicolumn{9}{|c|}{ Preschool children } \\
\hline \multirow[b]{2}{*}{ Condition* } & \multicolumn{4}{|c|}{ Arrow } & \multicolumn{4}{|c|}{ Shape } \\
\hline & \multirow{3}{*}{\multicolumn{2}{|c|}{$\begin{array}{c}\text { Nonpredictive } \\
N=14 \text { ( } 8 \text { males }) \\
\text { Mean: } 4 \text { yrs, } 7 \text { mos. } \\
\text { Range: } 3 \mathrm{y}, 5 \mathrm{~m} .-6 \mathrm{y}, 2 \mathrm{~m} . \\
8.5 \%\end{array}$}} & \multirow{3}{*}{\multicolumn{2}{|c|}{$\begin{array}{c}\text { Predictive } \\
N=15 \text { ( } 8 \text { males }) \\
\text { Mean: } 5 \text { yrs, } 3 \text { mos. } \\
\text { Range: } 4 \mathrm{y}, 11 \mathrm{~m} .-5 \mathrm{y}, 11 \mathrm{~m} . \\
7.58 \%\end{array}$}} & \multirow{3}{*}{\multicolumn{2}{|c|}{$\begin{array}{c}\text { Nonpredictive } \\
N=14 \text { ( } 7 \text { males }) \\
\text { Mean: } 4 \text { yrs, } 2 \text { mos. } \\
\text { Range: } 3 \mathrm{y}, 5 \mathrm{~m} .-5 \mathrm{y}, 4 \mathrm{~m} . \\
7.6 \%\end{array}$}} & \multirow{3}{*}{\multicolumn{2}{|c|}{$\begin{array}{c}\text { Predictive } \\
N=15 \text { ( } 9 \text { males }) \\
\text { Mean: } 4 \text { yrs, } 4 \mathrm{mos} . \\
\text { Range: } 3 \mathrm{y}, 4 \mathrm{~m} .-4 \mathrm{y}, 9 \mathrm{~m} . \\
9.19 \%\end{array}$}} \\
\hline Age & & & & & & & & \\
\hline \multirow[t]{2}{*}{$\%$ Error } & & & & & & & & \\
\hline & $M$ & $S D$ & $M$ & $S D$ & $M$ & $S D$ & $M$ & $S D$ \\
\hline \multicolumn{9}{|l|}{$100 \mathrm{~ms} \mathrm{SOA}$} \\
\hline Cued & 664 & 124 & 658 & 153 & 761 & 167 & 726 & 145 \\
\hline Uncued & 676 & 121 & 724 & 168 & 745 & 167 & 773 & 209 \\
\hline \multicolumn{9}{|l|}{$900 \mathrm{~ms}$ SOA } \\
\hline Cued & 597 & 90 & 585 & 94 & 642 & 135 & 644 & 128 \\
\hline Uncued & 622 & 106 & 618 & 146 & 692 & 206 & 646 & 149 \\
\hline \multicolumn{9}{|c|}{ Adults } \\
\hline & \multicolumn{4}{|c|}{ Arrow } & \multicolumn{4}{|c|}{ Shape } \\
\hline \multirow[t]{2}{*}{$\begin{array}{l}\text { Condition* } \\
\% \text { Error }\end{array}$} & \multicolumn{2}{|c|}{$\begin{array}{c}\text { Nonpredictive } \\
N=15(7 \text { males }) \\
.6 \%\end{array}$} & \multicolumn{2}{|c|}{$\begin{array}{c}\text { Predictive } \\
N=14(3 \text { males }) \\
1.2 \%\end{array}$} & \multicolumn{2}{|c|}{$\begin{array}{c}\text { Nonpredictive } \\
N=14 \text { (5 males) } \\
1.2 \%\end{array}$} & \multicolumn{2}{|c|}{$\begin{array}{c}\text { Predictive } \\
N=14(6 \text { males }) \\
0 \%\end{array}$} \\
\hline & $M$ & $S D$ & $M$ & $S D$ & $M$ & $S D$ & $M$ & SD \\
\hline \multicolumn{9}{|l|}{$100 \mathrm{~ms}$ SOA } \\
\hline Cued & 389 & 73 & 393 & 43 & 383 & 45 & 365 & 45 \\
\hline Uncued & 394 & 57 & 421 & 50 & 380 & 45 & 377 & 50 \\
\hline \multicolumn{9}{|l|}{$900 \mathrm{~ms}$ SOA } \\
\hline Cued & 338 & 48 & 329 & 37 & 351 & 38 & 327 & 33 \\
\hline Uncued & 349 & 53 & 364 & 27 & 347 & 41 & 341 & 32 \\
\hline
\end{tabular}

a $12^{\prime \prime}$ monitor set to black-and-white. Attaching an arrowhead and an arrowtail to both ends of a horizontal line created the central arrow cue, measuring $2.5^{\circ}$ of visual angle in length from the tip of the arrowhead to the end of the arrowtail. The central shape cues, a circle and a square, subtended $1.9^{\circ}$. Both cues were centered on the screen. The target was a black asterisk measuring $0.7^{\circ}$, appearing along the horizontal meridian with an eccentricity of $5^{\circ}$ as measured from central fixation to the target's center. The stimuli and sample trial sequence are illustrated in Figure 1.

For both age groups, the cue conditions (Nonpredictive Arrow (NA), Predictive Arrow (PA), Nonpredictive Shape (NS), Predictive Shape (PS)) were manipulated between participants. Stimulus onset asynchrony (SOA: 100, $900 \mathrm{~ms}$ ) and cue validity (cued, uncued) were manipulated within subjects. Each participant completed a total of 100 experimental trials, divided equally over two testing blocks except for two children who completed the second block after a break, and two children who completed only one block in the nonpredictive shape condition.

On each trial, there were two possible target locations, left and right of center. In the spatially nonpredictive cue

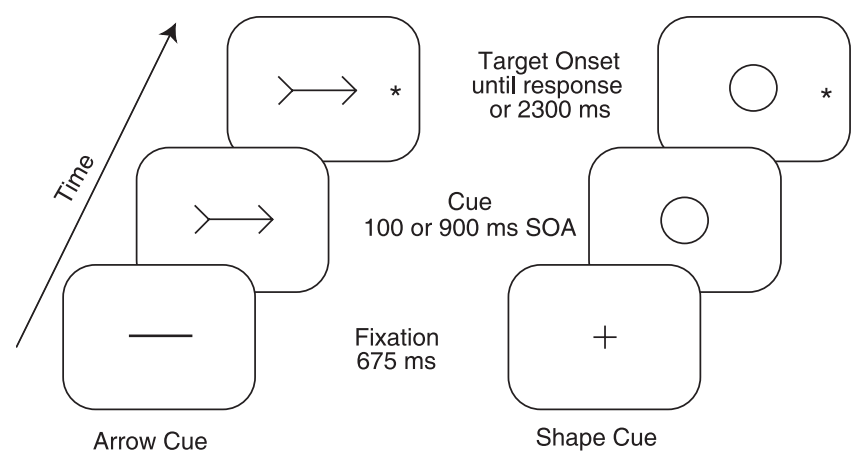

Figure 1 Illustration of stimuli and sample sequence of events. At the start of each trial, a straight line or a fixation point appeared on the screen for $675 \mathrm{~ms}$. Then, an arrow cue or a shape cue appeared on the screen. The target appeared centered across horizontal meridian either on the left or right side after 100 or $900 \mathrm{~ms}$. Both the cue and the target remained on the screen until response was made or for 2300 ms, whichever came first. Intertrial interval was 516 ms. The stimuli are not drawn to scale. 
conditions, the target appeared at the cued location 50\% of the time. In the spatially predictive cue conditions, the target appeared at the cued location $80 \%$ of the time. Arrow cues indicated the most likely target location by their direction. Shape cues indicated the most likely target location such that one shape predicted a target on the left while the other shape predicted a target on the right. The order shape-target position pairing was counterbalanced across participants.

\section{Procedure}

Participants were seated and centered with respect to an eye-level computer at a viewing distance of approximately $57 \mathrm{~cm}$. The start of every trial was signaled by a $675 \mathrm{~ms}$ presentation of a straight line (in the arrow condition) or a fixation cross (in the shape condition). The fixation was then replaced by a cue. An asterisk demanding a detection response appeared at one of the two target locations 100 or $900 \mathrm{~ms}$ after cue onset. Cue direction and SOA were varied equally and randomly. Both the cue and the target remained on the screen until a response was executed or $2300 \mathrm{~ms}$ had elapsed, whichever came first. The intertrial interval was $516 \mathrm{~ms}$. On approximately $6 \%$ of all the trials a target was not presented. These catch trials were dispersed randomly across all the trials. Reaction time (RT) to press the spacebar was measured from target onset.

All children were tested at a daycare facility. Two experimenters were present with the children at all times. One experimenter sat beside the child and ensured that each participant was responding with the proper key. The other experimenter was positioned behind the participant and recorded eye movements by observing participants' eyes in a mirror positioned above the computer screen.

After they had agreed to take part in the study, the procedure was explained to the children. In the nonpredictive cue conditions, they were informed that arrow or shape cues did not predict where the target would appear. In the predictive cue conditions, the children were informed about the predictive relationship between the cue and the target location. The experimenter ensured that all children understood instructions fully before commencing the experiment.

Undergraduate students were tested in the laboratory. Experimental procedure and instructions paralleled those employed with children, with the exception that only one experimenter was present in the testing room at all times.

\section{Results}

Incorrect key presses, timed-out responses, and false alarms counted as errors and were removed from the analysis. Response errors accounted for less than $6 \%$ of all trials and they were not analyzed further. Saccadic eye movements occurred on less than $5 \%$ of all the trials, ${ }^{1}$ agreeing with previous studies that eye movements are infrequent in target detection tasks (Posner, Snyder \& Davidson, 1980).

Median RTs were computed for each participant and were analyzed using an ANOVA with SOA and cue-target position validity included as within-subject factors and age group as a between-subjects factor. Significant interactions were followed by one-tailed $t$-tests capitalizing on evidence that RT is shorter at a valid compared to an invalid cue-target location (e.g. Posner, 1980).

Interparticipant mean RTs, eye movement rates, and error rates are presented in Table 1. Figure 2 illustrates average correct RTs for each age group and cue condition as a function of SOA and cue validity.

\section{Nonpredictive Arrow (NA)}

One can see in Figure 2A that for both age groups central nonpredictive arrow cues triggered reflexive orienting to the cued target location at both SOAs, with an overall RT decline occurring across SOA as is typical of a general warning signal effect (Mowrer, 1940). Main effects of age group $[F(1,27)=85.7, p<.0001]$, SOA $[F(1,27)=$ $26.2, p<.0001]$, and cue validity $[F(1,27)=4.33$, $p<.05$ ] were significant with no interactions [all $F \mathrm{~s}<1$ ].

\section{Predictive Arrow (PA)}

As illustrated in Figure 2B, both age groups also oriented attention to the location cued by a spatially predictive arrow, with faster RTs at the cued vs. uncued location for both SOAs. Main effects of age group $[F(1,27)=$ $65.6, p<.0001]$, SOA $[F(1,27)=23.1, p<.0001]$, and cue validity $[F(1,27)=25.4, p<.0001]$ were significant with no interactions [all $F \mathrm{~s}<1.2$, all $p \mathrm{~s}>.3$ ].

\section{Nonpredictive Shape (NS)}

In contrast to the nonpredictive arrow condition, the nonpredictive shape condition did not produce any reliable spatial orienting effects, as shown in Figure 2C. Mean RTs as a function of SOA, cue type, cue position, and

\footnotetext{
${ }^{1}$ By simultaneously observing participants' eye movements in the rearview mirror positioned above the computer monitor and the experimental sequence on the screen, the experimenter recorded whether, on every trial, an eye movement had occurred. Modified versions of this minimally invasive eye monitoring technique have been used successfully in past studies of attentional development (cf. Brodeur \& Enns, 1997) and adult attention (Friesen, Ristic \& Kingstone, 2004; Soto-Faraco, Morein-Zamir \& Kingstone, 2005). Although eye movements occurred infrequently (see Table 1), to ensure that our results reflected the deployment of covert orienting, the number of eye movements made either to the target or in anticipation of the target was included as a covariate in four preliminary ANCOVA analyses conducted for each cue condition separately with factors mirroring those reported in omnibus analyses in the Results section. As predicted, saccadic eye movements did not account for significant variation in the data [no $p$ s $<.05]$.
} 


\section{Spatial Predictiveness}

Nonpredictive

(A) Nonpredictive Arrow
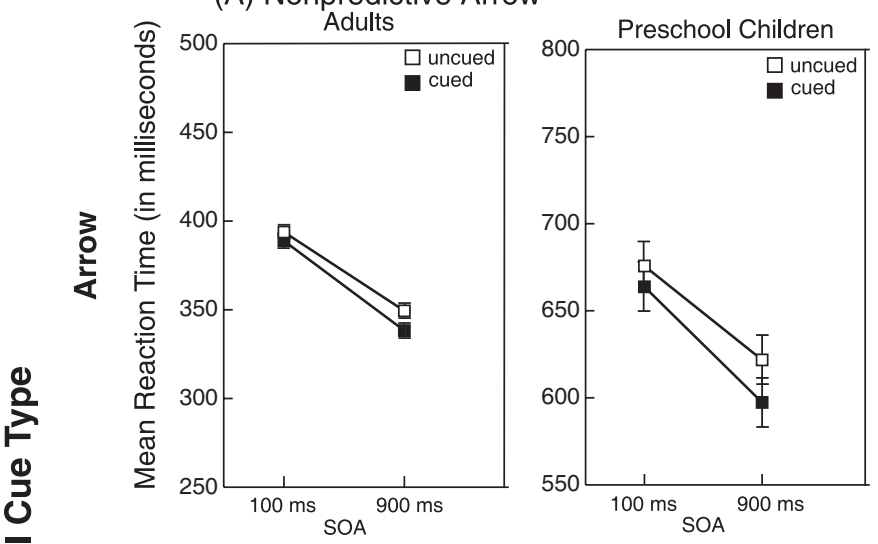

(C) Nonpredictive Shape
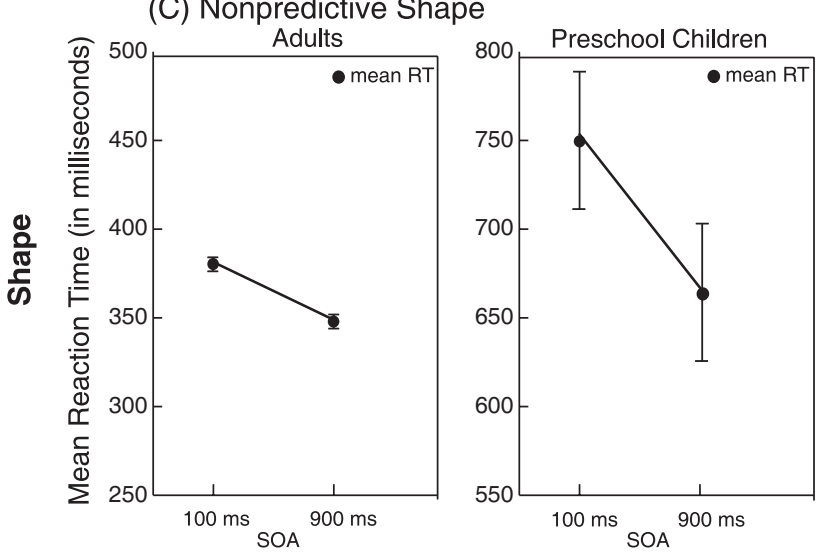

Predictive

(B) Predictive Arrow

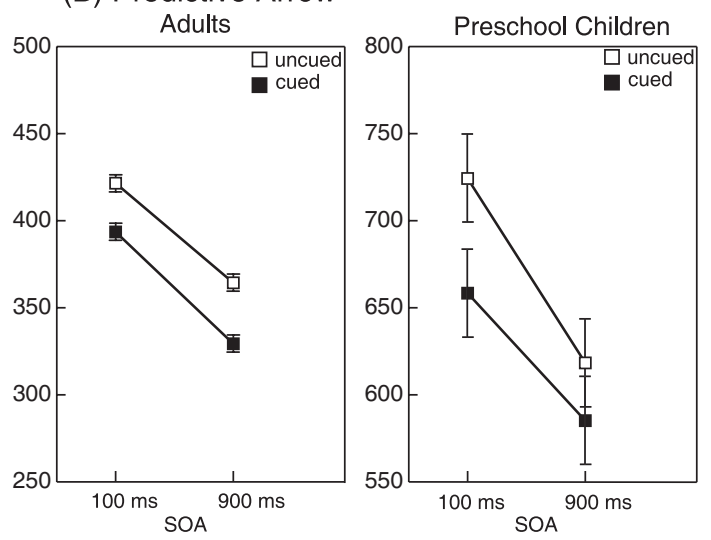

(D) Predictive Shape

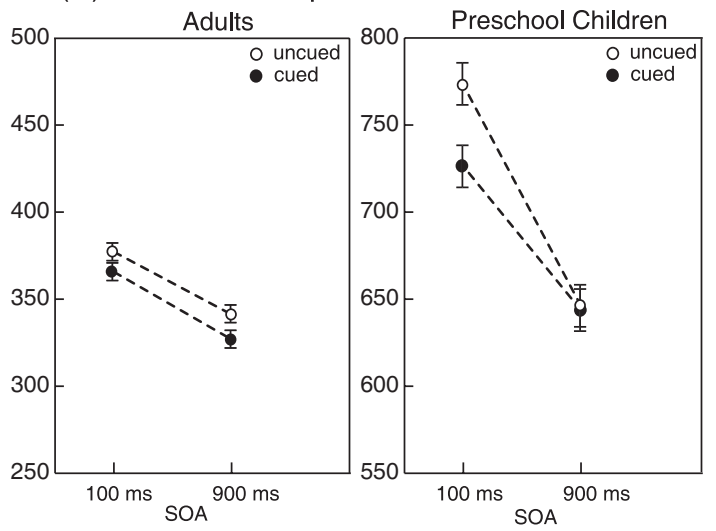

Figure 2 Mean response times (RTS) plotted as a function of SOA and cue validity for each age group and cue condition. Figures $2 A$ and $2 B$ show mean response times as a function of cue validity and SOA for nonpredictive and predictive directional arrow cues for both age groups. Figures $2 \mathrm{C}$ and $2 \mathrm{D}$ plot mean response times as a function of cue validity and SOA for nonpredictive and predictive central shape cues, with only an SOA effect plotted for nonpredictive shape condition. Error bars represent the standard error of the difference of the means.

age group were analyzed. Main effects of age group $[F(1,26)=109.2, p<.0001]$ and SOA $[F(1,26)=10.4$, $p<.01$ ] were significant with no interactions [all $F_{\mathrm{s}}<2.8$, all $p$ s $>$.1].

\section{Predictive Shape (PS)}

Given that spatial orienting did not occur for the nonpredictive shape cue condition, it follows that spatial attention effects in the predictive shape condition can be attributed to voluntary orienting. As shown in Figure 2D, when shape cues predicted where a target was likely to appear, adults were able to orient attention to the cued location and sustain their orienting across both SOAs. In contrast, preschool children were able to orient attention to the cued location at the shorter SOA but were unable to sustain volitional orienting out to the longer SOA. As a result, there were significant main effects of age group $[F(1,27)=70, p<.0001]$ and SOA $[F(1,27)=42.6, p<.0001]$ as well as an age group $\times$ SOA interaction $[F(1,27)=9.6, p<.01]$ reflecting a larger decline in RTs across the SOAs for children. Figure 2D also suggests an interaction between age group, cue validity, and SOA, which approached statistical significance $[F(1,27)=3, p<.09]$. Adults detected targets appearing at predicted locations $11.5 \mathrm{~ms}$ and $14.3 \mathrm{~ms}$ faster across the SOAs $[t(13)=2.73, p<.002 ; t(13)=1.85$, $p<.05$, respectively] while children were $47 \mathrm{~ms}$ faster to detect targets appearing at predicted locations but only at the short SOA of $100 \mathrm{~ms}[t(14)=1.72, p=.05$; $t(14)<1]$.

To examine potential developmental trends occurring between the ages of 3 and 6 in reflexive and volitional attention, each group of children was further divided into three age brackets ( $3-4$ years; $4-5$ years; $5-6$ years), a factor that was included as a between-subjects variable 

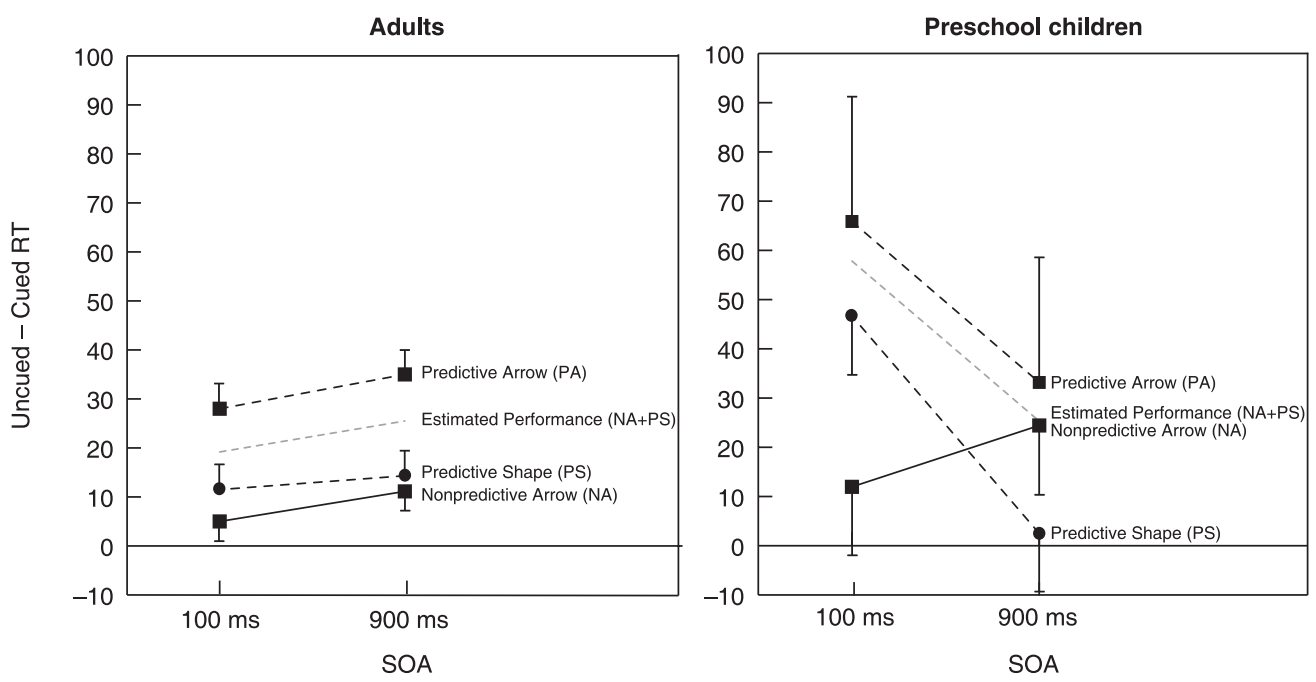

Figure 3 The magnitudes of attentional orienting for each age group and cue condition, computed as a difference between the uncued and cued RTS at each SOA interval. Left panel shows the magnitudes of attentional orienting for each cue condition for adults. Right panel illustrates the magnitudes of attentional orienting for each cue condition for children.

in the additional four ANOVAs conducted separately for each cue type condition. The results indicated that children's age did not reliably interact with any of the reported attentional effects [all $p \mathrm{~s}>.05$ ], thus demonstrating the stability of the attentional effects between 3 and 6 years of age for reflexive and volitional attention.

\section{Predictive arrow orienting and its component parts}

Do reflexive and volitional attention combine differently for adults and young children? Our data strongly suggest that they do. Figure 3 illustrates the mean magnitudes (uncued RT - cued RT) of attentional orienting elicited by a nonpredictive arrow (NA; reflexive orienting), a predictive shape (PS; volitional orienting), and a predictive arrow cue (PA; reflexive and volitional orienting). For adults, replicating Ristic and Kingstone (2006), the magnitude of the attention effect triggered by a predictive arrow cue is significantly larger than the sum of reflexive and volitional attention $[F(3,53)=4.7, p<.01]$. In contrast, for preschool children, the magnitude of the attention effect triggered by a predictive arrow cue is not reliably larger than the sum of reflexive and volitional attention $[F<1]$. That is, the data indicate that a predictive arrow cue elicits a superadditive interaction between reflexive and volitional orienting in adults, while the same cue elicits an additive combination between reflexive and volitional attention in children.

\section{Discussion}

Recent studies with adults have revealed that the predictive arrow task, which has been used for decades to measure the behavioral and neural markers of volitional orienting, has unwittingly confounded volitional and reflexive orienting. Instead of engaging volitional attention alone, this classic task engages both volitional and reflexive orienting, reflecting a superadditive interaction between the two (Ristic \& Kingstone, 2006; Olk, Cameron \& Kingstone, 2008).

We reasoned that the reported immaturity of "volitional' orienting measured with this confounded task in children younger than 8 years of age might be due to the fact that reflexive and volitional attention combine differently for preschool children and adults. To examine this possibility, we isolated reflexive orienting using a spatially nonpredictive arrow cue (NA condition) and volitional orienting using a predictive shape cue (PS condition; note that we confirmed that a nonpredictive shape (NS) does not engage reflexive attention). We also measured performance when volitional and reflexive orienting are confounded using the classic predictive arrow cue (PA condition). A group of children between 3 and 6 years old and a group of adults were tested.

For adults, and replicating previous reports, we found that the PA condition produced a superadditive orienting effect, i.e. the effect exceeded the sum of reflexive (NA) and volitional (PS) orienting.

For preschool children, again replicating previous reports, we found that reflexive orienting was comparable to adults' performance. However, volitional orienting was immature. The data from the PS condition, reflecting an isolated measure of volitional attention, indicated that volitional orienting was short-lived. The orienting effect emerged at an early $100 \mathrm{~ms}$ SOA and then rapidly dissipated by $900 \mathrm{~ms}$ SOA. This result demonstrates unequivocally, and for the first time, the immaturity of the isolated component of voluntary orienting in children between 3 and 6 years of age.

Another important difference between the two age groups was revealed in the magnitude of orienting observed in the PA task. Children's performance indicated a sum of reflexive (NA) and volitional (PS) processes, 
while in marked contrast adults' performance indicated an interaction between NA and PS processes. Thus, it is clear that in preschool children, just as in adults, the predictive arrow cue engages both volitional and reflexive orienting. However, in children, these two attentional effects combine independently, whereas in adults they interact. This conclusion suggests that past investigations of both typical (e.g. Brodeur et al., 1997) and atypical attentional development (e.g. Wainwright-Sharp \& Bryson, 1993; Pearson, Yaffee, Loveland \& Norton, 1995; Iarocci \& Burack, 2004) may have overestimated the contribution of voluntary orienting across the lifespan.

Thus, our data support the notion that children's immature performance on the classic arrow task does not reflect immaturity in volitional orienting alone but instead developmental changes in volitional attention and the immaturity in the combination of reflexive and volitional attention. Further, our result indicating that in preschool children reflexive orienting is adult-like but volitional orienting is not suggests that the change in how reflexive and volitional attention combine as a person ages depends primarily on the development of volitional orienting. In other words, reflexive and volitional attention do not interact when volitional attention is immature, but they do interact when volitional attention is fully developed, leading to the superadditive interaction observed in adults. Given that past studies indicate that the predictive arrow effect, and hence the interaction between reflexive and volitional orienting, becomes adult-like by the age of 8 , it is reasonable to predict that by 8 years of age, volitional orienting alone, as measured in the PS condition of the present study, will display adult-like characteristics.

Taken together, these conclusions highlight significant shortcomings in the classic cuing approach and carry important implications for the current understanding of attentional development and the brain areas that subserve that development. We discuss each point in turn.

In contrast to past investigations of reflexive attention development (e.g. Brodeur et al., 1997), our results from the nonpredictive arrow condition indicate that both preschool children and adults oriented reflexively in the direction of a nonpredictive arrow without producing inhibition of return (IOR), a behavioral effect that is tightly linked to oculomotor processes and which has traditionally been considered as an important behavioral marker of reflexive orienting (Posner \& Cohen, 1984).

Using a central cue instead of the peripheral one enabled us to unambiguously compare the effects of reflexive and volitional attention. As outlined in the introduction, in the past, behavioral comparisons between reflexive and volitional orienting typically involved reflexive attention being triggered by an abrupt onset of a peripheral nonpredictive stimulus and volitional attention being engaged by the presentation of a predictive central arrow stimulus. These procedural differences (a peripheral vs. a central cue) that were confounded with the type of attention being investigated (reflexive vs. volitional) compromised direct comparisons between reflexive and volitional orienting. Thus, it was never clear whether observed differences in performance should be attributed to differences in the cue conditions or differences in attentional orienting itself. The present data show that facilitative reflexive orienting comparable to those measured with peripheral cues could be obtained using a central cue (e.g. an arrow), therefore enabling direct comparisons between the two forms of attentional orienting (see also Hood, Willen \& Driver, 1998; Friesen \& Kingstone, 1998).

Finally, our results also offer insights into the brain areas that subserve attentional orienting in preschool years. Specifically, our data support the notion that the control of reflexive and volitional orienting in preschool children relies on the workings of the same cortical networks as those observed with adults. Adult neuroimaging data strongly suggest that the control of reflexive and volitional orienting depends on an involvement of two coordinated cortical networks: (1) a ventrolateral frontoparietal network, situated within the parietal cortex, which is recruited when observers orient reflexively; and (2) a dorsolateral frontoparietal network, situated within the frontal lobe, which is engaged when observers orient volitionally (Corbetta \& Shulman, 2002). The observed behavioral equivalence of reflexive orienting between children and adults as well as the absence of the subcortically mediated IOR suggest that the ventrolateral cortical network subserves reflexive orienting in preschool children. Conversely, the observed immaturity of volitional orienting in children supports the notion that the control of volitional attention in childhood depends on the involvement of the still underdeveloped dorsolateral network.

Because we also report age differences in the way reflexive and volitional attention combine, the brain mechanisms that mediate this interaction might also play a key role in determining attentional function in both childhood and adulthood. While the neural underpinnings of these interactions are still unexplored, one possible site is temporoparietal junction (TPJ), which appears to coordinate the operations of ventral and dorsal attentional networks (Friedrich, Egly, Rafal \& Beck, 1998; Corbetta \& Shulman, 2002). Thus, in addition to frontal lobe, full development of the TPJ might also play a key role in the development of the interaction between reflexive and volitional attention.

\section{Acknowledgements}

This research was supported by graduate fellowships awarded to JR from the Natural Sciences and Engineering Research Council (NSERC) and the Michael Smith Foundation for Health Research (MSFHR) and by grant support to AK from NSERC, MSFHR, Human Frontiers Science Project (HFSP) and Human Early Learning Partnership (HELP). We thank Jatinder (Bobby) Sidhu for his help with data collection. 


\section{References}

Berger, A., Jones, L., Rothbart, M.K., \& Posner, M.I. (2000). Computerized games to study the development of attention in childhood. Behavior Research Methods, Instruments, \& Computers, 32, 297-303.

Brodeur, D.A., \& Enns, J.T. (1997). Covert visual orienting across the lifespan. Canadian Journal of Experimental Psychology, 51, 20-35.

Brodeur, A.D., Trick, M.I., \& Enns, J.T. (1997). Selective attention over the lifespan. In A.J. Burack \& J.T. Enns (Eds.), Attention, development, and psychopathology (pp. 7497). New York: Guilford Press.

Corbetta, M., \& Shulman, G.L. (2002). Control of goal-directed and stimulus-driven attention in the brain. Nature Reviews Neuroscience, 3, 201-215.

Danziger, S., \& Kingstone, A. (1999). Unmasking the inhibition of return phenomenon. Perception \& Psychophysics, 61, 1024-1037.

Fan, J., McCandliss, B.D., Fossella, J., Flombaum, J.I., \& Posner, M.I. (2005). The activation of attentional networks. Neuroimage, 26, 471-479.

Fan, J., McCandliss, B.D., Sommer, T., Raz, A., \& Posner, M.I. (2002). Testing the efficiency and independence of attentional networks. Journal of Cognitive Neuroscience, 14, 340-347.

Friedrich, F.J., Egly, R., Rafal, R.D., \& Beck, D. (1998). Spatial attention deficits in humans: a comparison of superior parietal and temporal-parietal junction lesions. Neuropsychology, 12, 193-207.

Friesen, C.K., \& Kingstone, A. (1998). The eyes have it!: Reflexive orienting is triggered by nonpredictive gaze. Psychonomic Bulletin \& Review, 5, 490-495.

Friesen, C.K., Ristic, J., \& Kingstone, A. (2004). Attentional effects of counterpredictive gaze and arrow cues. Journal of Experimental Psychology: Human Perception and Performance, 30, 319-329.

Goldberg, M.C., Maurer, D., \& Lewis, T.L. (2001). Developmental changes in attention: the effects of endogenous cueing and of distractors. Developmental Science, 4, 209-219.

Hood, B.M., Willen, J.D., \& Driver, J. (1998). Adult's eyes trigger shifts of visual attention in human infants. Psychological Science, 9, 131-134.

Iarocci, G., \& Burack, J.A. (2004). Intact covert orienting to peripheral cues among children with autism. Journal of Autism and Developmental Disorders, 34, 257-264.

Johnson, M.H. (2002). The development of visual attention: a cognitive neuroscience perspective. In Y. Munakata \& R.O. Gilmore (Eds.), Brain development and cognition: A reader (pp. 134-150). Oxford: Blackwell.

Johnson, M.H. (2005). Developmental cognitive neuroscience. Cambridge, MA: Blackwell Publishers.
Johnson, M.H., Posner, M.I., \& Rothbart, M.K. (1991). Components of visual orienting in early infancy: contingency learning, anticipatory looking and disengaging. Journal of Cognitive Neuroscience, 3, 335-344.

Jonides, J. (1981). Voluntary versus automatic control over the mind's eye's movement. In J.B. Long \& A.D. Baddeley (Eds.), Attention and performance IX (pp. 187-203). Hillsdale, NJ: Erlbaum.

MacPhearson, A.C., Klein, R.M., \& Moore, C. (2003). Inhibition of return in children and adolescents. Journal of Experimental Child Psychology, 85, 337-351.

Mowrer, O.H. (1940). Preparatory set (expectancy) - Some methods of measurements. Psychological Review Monograph, 52 (Whole No. 233).

Olk, B., Cameron, B., \& Kingstone, A. (2008). Enhanced orienting effects: evidence for an interaction principle. Visual Cognition, 16, 979-1000.

Pearson, D.A., Yaffee, L.S., Loveland, K.A., \& Norton, A.M. (1995). Covert visual attention in children with attention deficit hyperactivity disorder: evidence for developmental immaturity? Development and Psychopathology, 7, 351-367.

Posner, M.I. (1980). Orienting of attention. Quarterly Journal of Experimental Psychology, 32, 3-25.

Posner, M.I., \& Cohen, Y. (1984). Components of visual orienting. In H. Bouma \& D.G. Bowhuis (Eds.), Attention and performance $X$ (pp. 531-556). Hillsdale, NJ: Erlbaum.

Posner, M.I., \& Petersen, S.E. (1990). The attention system of the human brain. Annual Review of Neuroscience, 13, 25-42.

Posner, M.I., Snyder, C.R.R., \& Davidson, B.J. (1980). Attention and detection of signals. Journal of Experimental Psychology: General, 109, 160-174.

Ristic, J., Friesen, C.K., \& Kingstone, A. (2002). Are eyes special? It depends on how you look at it. Psychonomic Bulletin \& Review, 9, 507-513.

Ristic, J., \& Kingstone, A. (2006). Attention to arrows: pointing in a new direction. Quarterly Journal of Experimental Psychology, 59, 1921-1931.

Soto-Faraco, S., Morein-Zamir, S., \& Kingstone, A. (2005). On audiovisual spatial synergy: the fragility of the phenomenon. Perception \& Psychophysics, 67, 444-457.

Tipples, J. (2002). Eye gaze is not unique: automatic orienting in response to noninformative arrows. Psychonomic Bulletin \& Review, 9, 314-318.

Wainwright-Sharp, J.A., \& Bryson, S.E. (1993). Visual orienting deficits in high functioning people with autism. Journal of Autism and Developmental Disorders, 23, 1-13.

Valenza, E., Simion, F., \& Umiltà, C. (1994). Inhibition of return in newborn infants. Infant Behavior and Development, 17, 293-302.

Received: 7 June 2007

Accepted: 6 March 2008 